\title{
The Problem of the Centre for Cubic Systems with Two Parallel Invariant Straight Lines and One Invariant Conic
}

\author{
Dumitru Cozma
}

\begin{abstract}
For cubic differential systems with two parallel invariant straight lines and at least one invariant conic it is proved that a singular point with pure imaginary eigenvalues (a weak focus) is a centre if and only if the first three Liapunov quantities $L_{j}, j=1,2,3$ vanish.

Mathematics Subject Classification (2000). Primary 34C05; Secondary 58F14. Keywords. Cubic differential systems, center-focus problem, invariant algebraic curves, integrability.
\end{abstract}

\section{Introduction}

In this paper we consider the cubic system of differential equations

$$
\begin{aligned}
& \dot{x}=y+a x^{2}+c x y+f y^{2}+k x^{3}+m x^{2} y+p x y^{2}+r y^{3} \equiv P(x, y), \\
& \dot{y}=-\left(x+g x^{2}+d x y+b y^{2}+s x^{3}+q x^{2} y+n x y^{2}+l y^{3}\right) \equiv-Q(x, y),
\end{aligned}
$$

in which all variables and coefficients are assumed to be real. The origin $O(0,0)$ is a singular point of a centre or a focus type for (1.1), i.e. a weak focus. The purpose of this paper is to find verifiable conditions for their distinctions.

It is known that the origin is a centre for system (1.1) if and only if it has in some neighborhood of $O(0,0)$ a $C^{\omega}$ nonconstant strong first integral $[4,16]$. Also, $O(0,0)$ is a centre if and only if $(1.1)$ has in some neighborhood of $O(0,0)$ a holomorphic integrating factor of the form $\mu=1+\sum \mu_{j}(x, y)[1]$.

There exists a formal power series $F(x, y)=\sum F_{j}(x, y)$ such that the rate of change of $F(x, y)$ along trajectories of (1.1) is a linear combination of polynomials $\left\{\left(x^{2}+y^{2}\right)^{j}\right\}_{j=2}^{\infty}: d F / d t=\sum_{j=2}^{\infty} L_{j-1}\left(x^{2}+y^{2}\right)^{j}$. Quantities $L_{j}, j=1,2, \ldots, \infty$ are polynomials in the coefficients of system (1.1) called the Liapunov quantities. The order of the weak focus $O(0,0)$ is $r$ if $L_{1}=L_{2}=\cdots=L_{r-1}=0$ but $L_{r} \neq 0$. 
The origin is a centre for (1.1) if and only if $L_{j}=0, j=1,2, \ldots, \infty$. By the Hilbert basis theorem there is $N$ such that $L_{j}=0$ for all $j$ if and only if $L_{j}=0$ for all $j \leq N$. It is only necessary to find a finite number of Liapunov quantities, though in any given case it is not known a priori how many are required. Thus, the set of points were we have a centre must be an algebraic set, which we call the centre variety.

The number $N$ is known only for quadratic systems $N=3[2,13]$ and for a cubic system with only homogeneous cubic nonlinearities $N=5[20,23]$. If the cubic system (1.1) contains both quadratic and cubic nonlinearities, the problem of the centre was solved only in some particular cases (see, for example, $[3,5,7,9$, 10, 17-19, 24]).

In this paper we solve the problem of the centre for cubic differential system (1.1) assuming that (1.1) has two parallel invariant straight lines and at least one invariant conic. The paper is organized as follows. The results concerning the relation between integrability, invariant algebraic curves and Liapunov quantities are presented in Section 2. In Section 3 we find nineteen sufficient series of conditions for the existence of two parallel invariant straight lines and at least one invariant conic. In Section 4 we obtain sufficient conditions for the existence of a centre and finally in Section 5 we give the proof of the main result.

\section{Invariant algebraic curves, Liapunov quantities, centre}

An algebraic curve $f(x, y)=0$ (real or complex) is said to be an invariant curve of system (1.1) if there exists a polynomial $K(x, y)$ such that $P \cdot \partial f / \partial x-Q \cdot \partial f / \partial y \equiv$ $K \cdot f$. The polynomial $K$ is called the cofactor of the invariant algebraic curve $f=0$. Let $f=f_{1}^{\alpha_{1}} f_{2}^{\alpha_{2}} \cdots f_{r}^{\alpha_{r}}$ be its factorization in irreducible factors over $\mathbb{C}[x, y]$, then $f=0$ is an invariant algebraic curve for (1.1) if and only if $f_{j}=0$ is an invariant algebraic curve for each $j=1, \ldots, r$. We shall consider only algebraic curves $f=0$ with $f$ irreducible.

If the cubic system (1.1) has sufficiently many invariant algebraic curves $f_{j}(x, y)=0, j=1, \ldots, q$, then in most cases a first integral (an integrating factor) can be constructed in the Darboux form [12]

$$
f_{1}^{\alpha_{1}} f_{2}^{\alpha_{2}} \cdots f_{q}^{\alpha_{q}} .
$$

The function (2.1), with $\alpha_{i} \in \mathbb{C}$ not all zero, is a first integral (an integrating factor) for (1.1) if and only if

$$
\begin{aligned}
\sum_{i=1}^{q} \alpha_{i} K_{i} & \equiv 0 \\
\left(\sum_{i=1}^{q} \alpha_{i} K_{i}\right. & \left.\equiv \frac{\partial Q}{\partial y}-\frac{\partial P}{\partial x}\right) .
\end{aligned}
$$


The method of Darboux turns out to be very useful and elegant one to prove integrability for some classes of systems depending on parameters. These last years, interesting results which relate algebraic solutions, Liapunov quantities and Darboux integrability have been published, see $[6,8,9,22]$.

Definition 2.1. We shall say that $\left(f_{j}, j=1, \ldots, M ; L=N\right)$ is $I L C$ ( $I$ - invariant algebraic curves, $L$ - Liapunov quantities, $C$ - centre) for (1.1), if the existence of $M$ algebraic curves $f_{j}(x, y)=0$ and the vanishing of the focal values $L_{\nu}, \nu=$ $1, \ldots, N$ implies the origin $O(0,0)$ to be a centre for $(1.1)$.

The ILC problem for cubic differential systems with invariant algebraic curves was considered in $[9-11,14,21]$. In these papers, the problem of the centre was completely solved for cubic systems with at least three invariant straight lines. The main results of these works are gathered in the following two theorems:

Theorem 2.2. $\left(f_{j}(x, y), f_{j}(0,0) \neq 0, j=1, \ldots, 4 ; L=1\right)$ is ILC for system (1.1).

Theorem 2.3. $\left(A_{j} x+B_{j} y+C_{j}, j=1, \ldots, 4 ; L=2\right)$ and $\left(A_{j} x+B_{j} y+C_{j}, j=\right.$ $1,2,3 ; L=7)$ are $I L C$ for cubic system (1.1).

\section{Conditions for the existence of two parallel invariant straight lines and at least one invariant conic}

Let the cubic system (1.1) have two parallel invariant straight lines $l_{1}, l_{2}, l_{1}|| l_{2}$ and at least one invariant conic. If $l_{1}$ and $l_{2}$ are real, then by a rotation of axes we can make them parallel to the axis of ordinates $(O y)$. Note that by a rotation of axes the linear part of the differential system (1.1) does not change the form. If $l_{1}$ and $l_{2}$ are complex, then the straight lines $\overline{l_{1}}, \overline{l_{2}}$ conjugate with $l_{1}$ and $l_{2}$ will be also invariant for (1.1) (the system (1.1) has real coefficients). As for (1.1) the problem of the centre with at least three invariant straight lines is solved, it remains to consider only the case when $l_{2} \equiv \overline{l_{1}}$. From $l_{1} \| \overline{l_{1}}$ it follows that $l_{1}$ looks as $1+A(x+B y)=0$, where $A$ is a complex number and $B$ is real. In this case, via a rotation of axes, it is also possible to make the straight lines $l_{1}$ and $l_{2}$ to be parallel to the axis $O y$. Later on this will be assumed to be fulfilled.

In order that the system (1.1) had two invariant straight lines $l_{1}, l_{2}$ parallel to the axis $O y$, it is necessary that the following coefficient conditions to be satisfied

$$
a=f=k=p=r=0, \quad m\left(c^{2}-4 m\right) \neq 0 .
$$

In this case the invariant straight lines $l_{1}$ and $l_{2}$ are

$$
l_{1,2} \equiv 1+\frac{c \pm \sqrt{c^{2}-4 m}}{2} x=0
$$

and have, respectively, the cofactors $K_{1,2}=y\left(2 m x+c \pm \sqrt{c^{2}-4 m}\right) / 2$.

We pass now to the problem of finding conditions for the existence of at least one invariant conic for (1.1). Let the conic curve be given by the equation

$$
f(x, y) \equiv a_{20} x^{2}+a_{11} x y+a_{02} y^{2}+a_{10} x+a_{01} y+1=0,
$$


with $\left(a_{20}, a_{11}, a_{02}\right) \neq 0$ and $a_{20}, a_{11}, a_{02}, a_{10}, a_{01} \in \mathbb{C}$.

For every conic curve (3.2) the following quantities

$$
I_{1}=a_{20}+a_{02}, \quad I_{2}=\frac{1}{4}\left|\begin{array}{cc}
2 a_{20} & a_{11} \\
a_{11} & 2 a_{02}
\end{array}\right|, \quad I_{3}=\frac{1}{8}\left|\begin{array}{ccc}
2 a_{20} & a_{11} & a_{10} \\
a_{11} & 2 a_{02} & a_{01} \\
a_{10} & a_{01} & 2
\end{array}\right|
$$

are invariants with respect to the translation and rotation of axes. These invariants will be taken into account classifying conics. A conic (3.2) is reducible into two straight lines if and only if $I_{3}=0$. In order to have an irreducible conic we will assume $I_{3} \neq 0$. If $I_{2}>0$, then (3.2) is an ellipse, if $I_{2}<0-$ a hyperbola and if $I_{2}=0-$ a parabola, see for instance [15].

The conic (3.2) is an invariant conic for (1.1) if and only if there exist numbers $c_{20}, c_{11}, c_{02}, c_{10}, c_{01} \in \mathbb{C}$ such that

$$
\frac{\partial f}{\partial x} \cdot P(x, y)-\frac{\partial f}{\partial y} \cdot Q(x, y) \equiv f(x, y)\left(c_{20} x^{2}+c_{11} x y+c_{02} y^{2}+c_{10} x+c_{01} y\right) \text {. }
$$

Identifying the coefficients of the monomials $x^{i} y^{j}$ in (3.3), we reduce this identity to a system of fourteen equations $\left\{F_{i j}=0\right\}$ for the unknowns $a_{i j}, c_{i j}$, $i+j=1,2$. We find that

$$
\begin{array}{ll}
c_{10}=-a_{01}, c_{01}=a_{10}, & c_{20}=a_{01} a_{10}-g a_{01}-a_{11}, \\
c_{11}=a_{01}^{2}-d a_{01}-a_{10}^{2}+c a_{10}-2 a_{02}+2 a_{20}, & c_{02}=a_{11}-b a_{01}-a_{01} a_{10},
\end{array}
$$

and $a_{20}, a_{11}, a_{02}, a_{10}, a_{01}$ are the solutions of the system

$$
\begin{aligned}
F_{40} \equiv & \left(a_{10}-g\right) a_{01} a_{20}-\left(a_{20}-s\right) a_{11}=0 \\
F_{31} \equiv & a_{01}^{2} a_{20}+\left(a_{10}-g\right) a_{01} a_{11}-d a_{01} a_{20}-2 a_{02}\left(a_{20}-s\right) \\
& -a_{11}^{2}+q a_{11}-a_{20}\left(a_{10}^{2}-c a_{10}-2 a_{20}+2 m\right)=0, \\
F_{22} \equiv & a_{01}^{2} a_{11}+\left(a_{10}-g\right) a_{01} a_{02}-d a_{01} a_{11}-\left(a_{10}+b\right) a_{01} a_{20} \\
& -3 a_{02} a_{11}+2 q a_{02}-a_{11}\left(a_{10}^{2}-c a_{10}-3 a_{20}+m-n\right)=0, \\
F_{13} \equiv & a_{01}^{2} a_{02}-d a_{01} a_{02}-\left(a_{10}+b\right) a_{01} a_{11}-2 a_{02}^{2}+a_{11}^{2}+l a_{11} \\
& -a_{02}\left(a_{10}^{2}-c a_{10}-2 a_{20}-2 n\right)=0, \\
F_{04} \equiv & a_{02}\left(a_{01} a_{10}+b a_{01}-a_{11}-2 l\right)=0, \\
F_{30} \equiv & \left(a_{10}-g\right)\left(a_{01} a_{10}-a_{11}\right)-\left(a_{20}-s\right) a_{01}=0, \\
F_{21} \equiv & \left(2 a_{10}-g\right) a_{01}^{2}-a_{01}\left(d a_{10}+2 a_{11}-q\right)-2\left(a_{10}-g\right) a_{02} \\
& -a_{10}^{3}+c a_{10}^{2}+3 a_{10} a_{20}-m a_{10}+d a_{11}-2 c a_{20}=0, \\
F_{12} \equiv & a_{01}^{3}-d a_{01}^{2}-3 a_{01} a_{02}-a_{01}\left(2 a_{10}^{2}+b a_{10}-c a_{10}-2 a_{20}-n\right) \\
& +2 d a_{02}+a_{11}\left(2 a_{10}+b-c\right)=0, \\
F_{03} \equiv & \left(a_{10}+b\right) a_{01}^{2}-a_{01}\left(a_{11}+l\right)-a_{02}\left(a_{10}+2 b\right)=0 .
\end{aligned}
$$

From $F_{04}=0$, we can see that either $a_{02}=0$ or $a_{11}=\left(a_{10}+b\right) a_{01}-2 l$. 


\subsection{Case $\mathrm{a}_{02}=0$}

In this case $F_{13} \equiv a_{11}\left(a_{01} a_{10}+b a_{01}-a_{11}-l\right)=0, F_{03} \equiv a_{01}\left(a_{01} a_{10}+b a_{01}-a_{11}-l\right)=$ 0 and we have the following possibilities:

3.1.1. $a_{01}=0, a_{11}=-l, l \neq 0$. In this case we obtain the following conditions 1)

$$
\begin{gathered}
a=f=k=p=r=0, \quad c=b+2 g, \quad d=(-2 b s-g n-4 g s) / l, \\
m=b g+g^{2}+n+3 s, \quad q=\left(-b g s-g^{2} s-l^{2}-2 n s-4 s^{2}\right) / l
\end{gathered}
$$

for the existence of a hyperbola $s x^{2}+g x-l x y+1=0$.

3.1.2. $a_{11}=0, a_{01} \neq 0$. In this case we get $a_{10}=g, a_{20}=s, g=-b, l=0$, $n=-2 m$ and $a_{01}$ can be found from the equations

$$
F_{21} \equiv q a_{01}-b m-b s-2 c s=0, \quad F_{12} \equiv a_{01}^{2}-d a_{01}-b^{2}-b c-2 m+2 s=0 .
$$

We obtain the following two series of conditions for the existence of conics:

2)

$$
a=f=k=l=p=q=r=0, \quad g=-b, \quad c=-b(m+s) /(2 s), \quad n=-2 m .
$$

The invariant conics are parabolas $s x^{2}-b x+a_{01} y+1=0$, where $a_{01}$ are found from the equation $2 s a_{01}^{2}-2 d s a_{01}+b^{2} m-b^{2} s-4 m s+4 s^{2}=0$.

3)

$$
\begin{aligned}
& a=f=k=l=p=r=0, \quad g=-b, \quad n=-2 m, \\
& d=\left[(b m+b s+2 c s)^{2}+\left(2 s-2 m-b c-b^{2}\right) q^{2}\right] /[q(b m+b s+2 c s)] .
\end{aligned}
$$

The invariant conic is a parabola $s x^{2}-b x+(b m+b s+2 c s) y / q+1=0$.

3.1.3. $\quad a_{11}=a_{01} a_{10}+b a_{01}-l \neq 0, a_{01} \neq 0$. In this case from $F_{30}=0$ we get

$$
a_{20}=\left(-b a_{01} a_{10}+b g a_{01}+s a_{01}+l a_{10}-g l\right) / a_{01} .
$$

Replacing $a_{20}$ in $F_{40}=0$ and factoring, we obtain $F_{40} \equiv u_{1} \cdot u_{2}=0$, where

$$
u_{1}=\left(b^{2}+b g+s\right) a_{01}^{2}-l(2 b+g) a_{01}+l^{2}, \quad u_{2}=a_{10}-g .
$$

Let $u_{1}=0$, then $I_{3}=0$ and the conic (3.2) is reducible. Next assume that $u_{1} \neq 0$ and $u_{2}=0$, i.e. $a_{10}=g$. The system of algebraic equations (3.4) becomes

$$
\begin{aligned}
F_{31} \equiv & \left(b^{2}+2 b g+g^{2}-s\right) a_{01}^{2}-(2 b l+b q-d s+2 g l+g q) a_{01} \\
& -c g s+g^{2} s+l^{2}+l q+2 m s-2 s^{2}=0, \\
F_{22} \equiv & (b+g) a_{01}^{3}-(b d+d g+l) a_{01}^{2}+\left(b c g-b g^{2}-b m\right. \\
& \left.+b n+2 b s+c g^{2}+d l-g^{3}-g m+g n+2 g s\right) a_{01}+l\left(g^{2}-c g\right. \\
& +m-n-3 s)=0, \\
F_{21} \equiv & (2 b+g) a_{01}^{2}-(b d+2 l+q) a_{01}-c g^{2}+2 c s+d l \\
& +g^{3}+g m-3 g s=0, \\
F_{12} \equiv & a_{01}^{3}-d a_{01}^{2}+\left(b^{2}-b c+2 b g+n+2 s\right) a_{01}+l(c-b-2 g)=0 .
\end{aligned}
$$


If $l=0$, then from $(3.5)$ we obtain $m=(c-b-g)(b+g), n=(c-b-2 g)(b+g)$ and $a_{01}$ can be found from the equations

$$
\begin{aligned}
& F_{21} \equiv(b d+d g-q) a_{01}+b^{2} g-b c g+3 b g^{2}-4 b s-c g^{2}+2 c s+2 g^{3}-5 g s=0, \\
& F_{12} \equiv a_{01}^{2}-d a_{01}-b g+c g-2 g^{2}+2 s=0 .
\end{aligned}
$$

We obtain the following two series of conditions for the existence of conics:

4)

$$
\begin{aligned}
& a=f=k=p=r=l=0, \quad m=(c-b-g)(b+g), \quad n=(c-b-2 g)(b+g), \\
& q=(b+g) d, \quad b^{2} g-b c g+3 b g^{2}-4 b s-c g^{2}+2 c s+2 g^{3}-5 g s=0,
\end{aligned}
$$

The invariant conics are hyperbolas $s x^{2}+(b+g) a_{01} x y+g x+a_{01} y+1=0$, where $a_{01}$ are the solutions of the equation $a_{01}^{2}-d a_{01}-b g+c g-2 g^{2}+2 s=0$.

5)

$$
\begin{aligned}
& a=f=k=p=r=l=0, \quad m=(c-b-g)(b+g), \quad n=(c-b-2 g)(b+g), \\
& \left(b^{2} g-b c g+b d^{2}+3 b g^{2}-4 b s-c g^{2}+2 c s+d^{2} g-d q+2 g^{3}-5 g s\right) \\
& \quad \cdot\left(b^{2} g-b c g+3 b g^{2}-4 b s-c g^{2}+2 c s+2 g^{3}-5 g s\right) \\
& \quad+\left(c g-b g-2 g^{2}+2 s\right)(b d+d g-q)^{2}=0 .
\end{aligned}
$$

The invariant conic is $s x^{2}+(b+g) a_{01} x y+g x+a_{01} y+1=0$, where

$$
a_{01}=\left(-b^{2} g+b c g-3 b g^{2}+4 b s+c g^{2}-2 c s-2 g^{3}+5 g s\right) /(b d+d g-q) .
$$

If $l \neq 0$, then we reduce the equation $F_{31}=0$ by $q$ from $F_{21}=0$, the equations $\left\{F_{31}=0, F_{22}=0, F_{21}=0\right\}$ by $s$ from $F_{12}=0$, the equations $\left\{F_{31}=0, F_{21}=\right.$ $\left.0, F_{12}=0\right\}$ by $n$ from $F_{22}=0$ and the equations $\left\{F_{22}=0, F_{21}=0\right\}$ by $d$ from $F_{31}=0$. The system (3.5) becomes:

$$
\begin{aligned}
F_{31} \equiv & l a_{01}^{2}+\left(2 b^{2} c-2 b^{3}-6 b^{2} g+4 b c g-6 b g^{2}-2 b m+2 c g^{2}\right. \\
& \left.-d l-2 g^{3}-2 g m\right) a_{01}+(2 b+g)(b-c+2 g) l=0 \\
F_{22} \equiv & \left(3 b^{2}-3 b c+7 b g-3 c g+4 g^{2}+2 m+n\right) a_{01} \\
& +3 l(c-b-2 g)=0 \\
F_{21} \equiv & l(b+g) a_{01}^{3}+\left[\left(b^{2}-b c+2 b g-c g+g^{2}+m\right)(2 b-2 c+3 g)\right. \\
& \left.\cdot(b+g)-l^{2}-l q\right] a_{01}^{2}-l\left(4 b^{2}-4 b c+9 b g-4 c g+5 g^{2}+2 m\right) \\
& \cdot(b-c+2 g) a_{01}+2(b-c+2 g)^{2} l^{2}=0, \\
F_{12} \equiv & \left(b^{2}-b c+2 b g-c g+g^{2}+m\right)(b+g) a_{01}^{2}-l[(2 b-2 c+3 g) \\
& \cdot(b+g)+m-s] a_{01}+l^{2}(b-c+2 g)=0 .
\end{aligned}
$$

Now consider the equation $F_{22}=0$. If $3 b^{2}-3 b c+7 b g-3 c g+4 g^{2}+2 m+n=0$, then $c=b+2 g$ and we obtain the following conditions for the existence of a conic 
6)

$$
\begin{aligned}
& a=f=k=p=r=0, \quad c=b+2 g, \quad n=2\left(b g+g^{2}-m\right), \\
& q=\left[\left(m-b g-g^{2}\right)(2 b+g)(b+g)+(b d+d g-l) l\right] / l, \\
& s=\left[2\left(m-b g-g^{2}\right)(b+g)^{2}+(b d+d g-l) l\right]\left(b g+g^{2}-m\right) / l^{2} .
\end{aligned}
$$

The invariant conic is a hyperbola $s x^{2}+\left((b+g) a_{01}-l\right) x y+g x+a_{01} y+1=0$, where $a_{01}=\left[2\left(m-b g-g^{2}\right)(b+g)+d l\right] / l$.

Denote $\gamma=3 b^{2}-3 b c+7 b g-3 c g+4 g^{2}+2 m+n$ and let $\gamma \neq 0$, then from $F_{22}=0$ we get $a_{01}=(3 l(b-c+2 g)) / \gamma$. Replacing $a_{01}$ in (3.6), we find the following series of conditions for the existence of a conic

7)

$$
\begin{aligned}
a= & f=k=p=r=0, \quad s=\left[\left(2 b g+2 g^{2}-2 m-n\right)\left(b g+g^{2}-m+n\right)\right] /(3 \gamma), \\
d= & {\left[\left(3 b c g-b^{2} g-3 b g^{2}-2 b m+2 b n+3 c g^{2}-2 g^{3}-4 g m+g n\right) \gamma+\right.} \\
& \left.+9(b-c+2 g) l^{2}\right] /(3 l \gamma), \\
q= & {\left[\left(m-n-b g-g^{2}\right)\left(b g+g^{2}-4 m-2 n\right) \gamma+9 l^{2}\left(2 b g+2 g^{2}-2 m-n\right)\right] /(9 l \gamma) . }
\end{aligned}
$$

The invariant conic is a hyperbola $s x^{2}+\left((b+g) a_{01}-l\right) x y+g x+a_{01} y+1=0$, where $a_{01}=(3 l(b-c+2 g)) / \gamma$.

\subsection{Case $\mathrm{a}_{02} \neq 0$}

In this case from $F_{04}=0$ of (3.4) we have $a_{11}=\left(a_{10}+b\right) a_{01}-2 l$ and replace in the remaining equations of (3.4). We consider the following possibilities:

3.2.1. $\quad a_{01}=0$. Then from $F_{03}=0$ we find $a_{10}=-2 b$ and

$$
F_{40} \equiv l\left(a_{20}-s\right)=0, \quad F_{30} \equiv l(2 b+g)=0 .
$$

If $l=0$, then $q=d=0, a_{20}=a_{02}+2 b^{2}+b c-n$ and the system of equations (3.4) becomes:

$$
\begin{aligned}
& F_{31} \equiv(m+n-s) a_{02}+2 b^{2} m+2 b^{2} n+b c m+b c n-m n-n^{2}=0, \\
& F_{21} \equiv(b+c-g) a_{02}+2 b^{3}+3 b^{2} c+b c^{2}-b m-3 b n-c n=0 .
\end{aligned}
$$

Solving (3.7) for $s$ and $g$ we obtain the following series of conditions for the existence of a conic

8)

$$
\begin{aligned}
& a=f=k=p=r=d=l=q=0, \\
& g=\left(b a_{02}+c a_{02}+2 b^{3}+3 b^{2} c+b c^{2}-b m-3 b n-c n\right) / a_{02}, \\
& s=\left(m a_{02}+n a_{02}+2 b^{2} m+2 b^{2} n+b c m+b c n-m n-n^{2}\right) / a_{02} .
\end{aligned}
$$

In this case we have a one-parametric family of conics

$$
\left(a_{02}+2 b^{2}+b c-n\right) x^{2}+a_{02} y^{2}-2 b x+1=0 .
$$

If $l \neq 0$, then $a_{20}=s, g=-2 b$ and from (3.4) we obtain the following two series of conditions for the existence of a conic: 
9)

$$
\begin{aligned}
& a=f=k=p=r=0, \quad g=-2 b, \quad d=(-s(3 b+c)) / l, \quad m=-2 b(2 b+c), \\
& n=\left(2 b^{2} s+b c s+l^{2}-2 s^{2}\right) / s, \quad q=\left(2 b^{2} s+b c s-2 l^{2}+s^{2}\right) / l .
\end{aligned}
$$

The invariant conic is a parabola $s x^{2}-2 l x y+l^{2} y^{2} / s-2 b x+1=0$.

10)

$$
\begin{aligned}
& a=f=k=p=r=0, \quad g=-2 b, \quad c=-2 b, \quad d=(b(m-s)) / l, \\
& q=\left(s^{2}-2 l^{2}-m s\right) / l, \quad n=\left(m^{2}-l^{2}-3 m s+2 s^{2}\right) /(m-s) .
\end{aligned}
$$

The invariant conic is $s x^{2}-2 l x y-l^{2} y^{2} /(m-s)-2 b x+1=0$.

3.2.2. $\quad a_{01} \neq 0$. In this case from $F_{30}=0$ of (3.4) we get

$$
a_{20}=\left(-b a_{01} a_{10}+b g a_{01}+s a_{01}+2 l a_{10}-2 g l\right) / a_{01} .
$$

Replacing $a_{20}$ in $F_{40}=0$ and factoring, we obtain $F_{40} \equiv v_{1} \cdot v_{2}=0$, where

$$
v_{1}=a_{10}-g, \quad v_{2}=\left(b^{2}+b g+s\right) a_{01}^{2}-2 l(2 b+g) a_{01}+4 l^{2} .
$$

We divide the investigation into two cases:

3.2.2.1. $v_{1}=0$, i.e. $a_{10}=g$. We express $n$ from $F_{13}=0, q$ from $F_{22}=0$ and replace in $F_{31}=0$. Factoring we obtain $F_{31} \equiv i_{1} \cdot i_{2}=0$, where

$$
\begin{aligned}
& i_{1}=a_{01}^{2} a_{02}-d a_{01} a_{02}+l(b+g) a_{01}+\left(c g-g^{2}-2 m+2 s\right) a_{02}-2 l^{2}, \\
& i_{2}=(b+g)^{2} a_{01}^{2}-4 l(b+g) a_{01}-4 s a_{02}+4 l^{2} .
\end{aligned}
$$

Let $i_{1}=0$ and consider the equation $F_{03} \equiv(2 b+g) a_{02}-l a_{01}=0$ of (3.4). If $g=-2 b$, then $l=0$ and we obtain

11)

$$
\begin{array}{ll}
a=f=k=p=r=l=0, & g=-2 b, \quad c=-2 b, \quad q=-b a_{01}, \\
s=\left(-a_{01}^{2}+d a_{01}+2 m\right) / 2, & n=\left(b^{2} a_{01}+m a_{01}-2 d m\right) /\left(2\left(d-a_{01}\right)\right) .
\end{array}
$$

The invariant conic is $\left(a_{01}^{2}-d a_{01}-2 m\right) x^{2} / 2+b a_{01} x y+\left(\left(b^{2}-m\right) a_{01}\right) y^{2} /\left(2\left(a_{01}-\right.\right.$ d) $)+2 b x-a_{01} y-1=0$.

12)

$$
\text { If } i_{1}=0 \text { and } g+2 b \neq 0 \text {, then we obtain }
$$

$$
\begin{aligned}
a= & f=k=p=r=0, \quad c=2(b+g), \\
n= & {\left[-l a_{01}^{2}+\left(2 b^{3}+3 b^{2} g+b g^{2}+2 d l\right) a_{01}-2 l\left(2 b^{2}+3 b g+g^{2}\right)\right] /\left(a_{01}(2 b+g)\right), } \\
m= & {\left[2 l a_{01}^{2}+\left(2 b^{3}+5 b^{2} g+4 b g^{2}-2 d l+g^{3}\right) a_{01}-2 b l(2 b+g)\right] /\left(a_{01}(2 b+g)\right), } \\
q= & {\left[g a_{01}^{3}+d(2 b+g) a_{01}^{2}-\left(2 b^{2} g+b g^{2}+4 d l\right) a_{01}+2 g l(2 b+g)\right] /\left(2 a_{01}^{2}\right), } \\
s= & {\left[(-2 b-g) a_{01}^{3}+(2 b d+d g+4 l) a_{01}^{2}+\left(-2 b^{2} g-b g^{2}-4 d l\right) a_{01}\right.} \\
& +2 g l(2 b+g)] /\left(2 a_{01}(2 b+g)\right) .
\end{aligned}
$$

The invariant conic is $s x^{2}+\left(b a_{01}+g a_{01}-2 l\right) x y+\left(l a_{01} y^{2}\right) /(2 b+g)+g x+a_{01} y+1=0$. 
Let now $i_{1} \neq 0, i_{2}=0$ and consider the equation $F_{03} \equiv(2 b+g) a_{02}-l a_{01}=0$ of (3.4). If $g=-2 b$, then $l=0$ and we obtain

13)

$$
\begin{aligned}
a & =f=k=p=r=l=0, \quad g=-2 b, \quad s=\left(-a_{01}^{2} b^{2}\right) /\left(4\left(2 b^{2}+b c-n\right)\right) \\
d & =\left[a_{01}\left(3 b^{2}+2 b c-2 n\right)\right] /\left(2\left(2 b^{2}+b c-n\right)\right), \quad m=2 b(-2 b-c) \\
q & =\left[a_{01} b\left(-6 b^{2}-3 b c+2 n\right)\right] /\left(2\left(2 b^{2}+b c-n\right)\right) .
\end{aligned}
$$

The invariant conic is a parabola $s x^{2}-b a_{01} x y-\left(2 b^{2}+b c-n\right) y^{2}-2 b x+a_{01} y+1=0$.

If $i_{2}=0$ and $g+2 b \neq 0$, then we get

14)

$$
\begin{aligned}
a= & f=k=p=r=0, \quad m=2(c-2 b-2 g)(b+g), \\
d= & {\left[2 l a_{01}^{2}+(2 b+g)(b+g)^{2} a_{01}+2(2 b+g)(b-c+2 g) l\right] /\left(2 l a_{01}\right), } \\
n= & {\left[l a_{01}^{2}+(4 b-c+3 g)(2 b+g)(b+g) a_{01}-2(2 b+g)^{2} l\right] /\left(a_{01}(2 b+g)\right), } \\
s= & {\left[\left((b+g) a_{01}-2 l\right)^{2}(2 b+g)\right] /\left(4 l a_{01}\right), } \\
q= & {\left[\left(4 l a_{01}^{2}-(2 b-2 c+3 g)(2 b+g)(b+g) a_{01}-2 l(2 b+g)^{2}\right)\right.} \\
& \left.\cdot\left(b a_{01}+g a_{01}-2 l\right)\right] /\left(4 l a_{01}^{2}\right) .
\end{aligned}
$$

The invariant conic is a parabola

$$
s x^{2}+\left((b+g) a_{01}-2 l\right) x y+\left(a_{01} l y^{2}\right) /(2 b+g)+g x+a_{01} y+1=0 .
$$

3.2.2.2. $v_{1} \neq 0, v_{2}=0$. From $F_{03} \equiv l a_{01}-a_{02} a_{10}-2 b a_{02}=0$ of (3.4) we get $a_{10}=\left(l a_{01}-2 b a_{02}\right) / a_{02}$ and replace in the remaining equations of (3.4).

Reduce the equations $\left\{F_{31}=0, F_{22}=0, F_{13}=0, F_{21}=0, F_{12}=0\right\}$ of (3.4) by $s$ from $v_{2}=0$. Express $q$ from $F_{21}=0, n$ from $F_{13}=0, d$ from $F_{12}=0$ and replace in $\left\{F_{31}=0, F_{22}=0\right\}$. Next reduce the equation $F_{31}=0$ by $g$ from $F_{22}=0$.

Denote $\sqrt{c^{2}-4 m}=u$, then $m=\left(c^{2}-u^{2}\right) / 4$. If $c^{2}-4 m>0$ then the invariant straight lines (3.1) are real, conversely if $c^{2}-4 m<0$, they are complex. Replacing this in $F_{31}=0$ and factoring, we obtain $F_{31} \equiv j_{1} \cdot j_{2} \cdot j_{3}=0$, where

$$
j_{1,2}=\left(2 b a_{01}+c a_{01} \mp u a_{01}-4 l\right) a_{02}-2 l a_{01}^{2}, \quad j_{3}=\left(b a_{01}-2 l\right) a_{02}-l a_{01}^{2} .
$$

Assume that $j_{1,2}=0$. In this case we get $a_{02}=\left(2 l a_{01}^{2}\right) /\left(2 b a_{01}+c a_{01} \mp u a_{01}-\right.$ $4 l)$. Consider the equation $v_{2}=0:\left(b^{2}+b g+s\right) a_{01}^{2}-2 l(2 b+g) a_{01}+4 l^{2}=0$.

If $b^{2}+b g+s=0$, then $a_{01}=(2 l) /(2 b+g)$ and we obtain the following series of conditions for the existence of a conic: 
15)

$$
\begin{aligned}
a= & f=k=p=r=0, \quad m=\left(c^{2}-u^{2}\right) / 4, \quad s=-b(b+g), \\
d= & {\left[\left(82 b^{2}-11 b c+82 b g \pm 33 b u-5 c g \mp 3 c u+20 g^{2} \pm 17 g u+3 u^{2}\right) l\right] / } \\
& {[(8 b-c+4 g \pm u)(4 b+2 g \pm u)(2 b+g)], } \\
q= & {\left[-\left(10 b^{2}+b c-2 b g \pm 3 b u+c g-4 g^{2} \mp g u\right) l\right] /[2(4 b+2 g \pm u)(2 b+g)], } \\
n= & {\left[16 b^{3}-38 b^{2} c+72 b^{2} g \pm 30 b^{2} u+5 b c^{2}-50 b c g \mp 16 b c u+64 b g^{2}\right.} \\
& \pm 46 b g u+11 b u^{2}+3 c^{2} g \pm c^{2} u-16 c g^{2} \mp 10 c g u-2 c u^{2}+16 g^{3} \\
& \left. \pm 16 g^{2} u+7 g u^{2} \pm u^{3}\right] /[(2(6 b-c+4 g \pm u)], \\
l^{2}= & {\left[(8 b-c+4 g \pm u)(4 b+2 g \pm u)(2 b-c+2 g \pm u)(2 b+g)^{2}\right] / } \\
& {[4(4 g \pm u-c+6 b)] . }
\end{aligned}
$$

The invariant conic is $(3.2)$, where

$$
\begin{aligned}
& a_{10}=(c-6 b-2 g \mp u) / 2, \quad a_{01}=(2 l) /(2 b+g), \\
& a_{20}=((c-8 b-4 g \mp u)(b+g)) / 2, \quad a_{11}=(l(c-8 b-4 g \mp u)) /(2 b+g), \\
& a_{02}=((c-8 b-4 g \mp u)(4 b+2 g \pm u)(2 b+g)) /(4 g \pm u-c+6 b) .
\end{aligned}
$$

If $b^{2}+b g+s \neq 0$, then denote $\sqrt{g^{2}-4 s}=v$. We express $s=\left(g^{2}-v^{2}\right) / 4$ and replacing in $v_{2}=0$ one obtain $v_{2}=h_{1} \cdot h_{2}$, where $h_{1}=(2 b+g+v) a_{01}-4 l$ and $h_{2}=(2 b+g-v) a_{01}-4 l$.

Let $h_{1}=0$, then $a_{01}=(4 l) /(2 b+g+v)$ and we obtain the following series of conditions for the existence of a conic:

16)

$$
\begin{aligned}
a= & f=k=p=r=0, \quad m=\left(c^{2}-u^{2}\right) / 4, \quad s=\left(g^{2}-v^{2}\right) / 4, \\
d= & {\left[\left(48 b^{2}-12 b c+48 b g \pm 36 b u+40 b v-5 c g \mp 6 c u-5 c v+11 g^{2}\right.\right.} \\
& \left.\left. \pm 19 g u+20 g v+6 u^{2} \pm 15 u v+9 v^{2}\right) l\right] /[(4 b-c+2 g \pm u+2 v) \\
& \cdot(2 b+g \pm u+v)(2 b+g+v)], \\
n= & {\left[\left(16 b^{2} c-24 b^{2} g \mp 16 b^{2} u-8 b^{2} v-4 b c^{2}+28 b c g \pm 16 b c u+20 b c v\right.\right.} \\
& -28 b g^{2} \mp 32 b g u-32 b g v-12 b u^{2} \mp 16 b u v-4 b v^{2}-3 c^{2} g \mp 2 c^{2} u \\
& -3 c^{2} v+11 c g^{2} \pm 12 c g u+16 c g v+4 c u^{2} \pm 8 c u v+5 c v^{2}-8 g^{3} \\
& \left.\left.\mp 13 g^{2} u-16 g^{2} v-9 g u^{2} \mp 16 g u v-8 g v^{2} \mp 2 u^{3}-5 u^{2} v \mp 3 u v^{2}\right)\right] / \\
& {[4(c-4 b-3 g \mp u-v)], } \\
q= & {\left[\left(12 b g-4 b v-c g-c v+7 g^{2} \pm 5 g u+4 g v \mp 3 u v-3 v^{2}\right) l\right] / } \\
& {[2(2 b+g \pm u+v)(2 b+g+v)], } \\
l^{2}= & {\left[(4 b-c+2 g \pm u+2 v)(2 b+g \pm u+v)(2 b+g+v)^{2}(c-g\right.} \\
& \mp u-v)] /[16(c-4 b-3 g \mp u-v)] .
\end{aligned}
$$


The invariant conic is $(3.2)$, where

$$
\begin{aligned}
& a_{10}=(c-4 b-g \mp u-v) / 2, \quad a_{20}=[(c-4 b-2 g \mp u-2 v)(g+v)] / 4, \\
& a_{01}=(4 l) /(2 b+g+v), \quad a_{11}=[2 l(c-4 b-2 g \mp u-2 v)] /(2 b+g+v), \\
& a_{02}=\left(8 l^{2}\right) /[(c-g \mp u-v)(2 b+g+v)] .
\end{aligned}
$$

Let $h_{1} \neq 0, h_{2}=0$, then $a_{01}=(4 l) /(2 b+g-v)$ and we obtain the following series of conditions for the existence of a conic:

17)

$$
\begin{aligned}
a= & f=k=p=r=0, \quad m=\left(c^{2}-u^{2}\right) / 4, \quad s=\left(g^{2}-v^{2}\right) / 4, \\
d= & {\left[\left(48 b^{2}-12 b c+48 b g \pm 36 b u-40 b v-5 c g \mp 6 c u+5 c v+11 g^{2}\right.\right.} \\
& \left.\left. \pm 19 g u-20 g v+6 u^{2} \mp 15 u v+9 v^{2}\right) l\right] /[(4 b-c+2 g \pm u-2 v) \\
& \cdot(2 b+g \pm u-v)(2 b+g-v)], \\
n= & {\left[\left(16 b^{2} c-24 b^{2} g \mp 16 b^{2} u+8 b^{2} v-4 b c^{2}+28 b c g \pm 16 b c u-20 b c v\right.\right.} \\
& -28 b g^{2} \mp 32 b g u+32 b g v-12 b u^{2} \pm 16 b u v-4 b v^{2}-3 c^{2} g \mp 2 c^{2} u \\
& +3 c^{2} v+11 c g^{2} \pm 12 c g u-16 c g v+4 c u^{2} \mp 8 c u v+5 c v^{2}-8 g^{3} \\
& \left.\left.\mp 13 g^{2} u+16 g^{2} v-9 g u^{2} \pm 16 g u v-8 g v^{2} \mp 2 u^{3}+5 u^{2} v \mp 3 u v^{2}\right)\right] / \\
& {[4(c-4 b-3 g \mp u+v)], } \\
q= & {\left[\left(12 b g+4 b v-c g+c v+7 g^{2} \pm 5 g u-4 g v \pm 3 u v-3 v^{2}\right) l\right] / } \\
& {[2(2 b+g \pm u-v)(2 b+g-v)], } \\
l^{2}= & {\left[(4 b-c+2 g \pm u-2 v)(2 b+g \pm u-v)(2 b+g-v)^{2}(c-g\right.} \\
& \mp u+v)] /[16(c-4 b-3 g \mp u+v)] .
\end{aligned}
$$

The invariant conic is $(3.2)$, where

$$
\begin{aligned}
& a_{10}=(c-4 b-g \mp u+v) / 2, \quad a_{20}=[(c-4 b-2 g \mp u+2 v)(g-v)] / 4, \\
& a_{01}=(4 l) /(2 b+g-v), \quad a_{11}=[2 l(c-4 b-2 g \mp u+2 v)] /(2 b+g-v), \\
& a_{02}=\left(8 l^{2}\right) /[(c-g \mp u+v)(2 b+g-v)] .
\end{aligned}
$$

Assume now that $j_{1,2} \neq 0$ and $j_{3}=0$. Then we get $a_{02}=\left(l a_{01}^{2}\right) /\left(b a_{01}-2 l\right)$. Consider the equation $v_{2}=0$ :

$$
\left(b^{2}+b g+s\right) a_{01}^{2}-2 l(2 b+g) a_{01}+4 l^{2}=0 .
$$

If $b^{2}+b g+s=0$, then $a_{01}=(2 l) /(2 b+g)$ and we obtain the following series of conditions for the existence of a conic: 
18)

$$
\begin{aligned}
a= & f=k=p=r=0, \quad m=\left(c^{2}-u^{2}\right) / 4, \quad s=-b(b+g), \\
d= & {\left[2\left(82 b^{2}+22 b c+82 b g+c^{2}+12 c g+20 g^{2}-u^{2}\right) l\right] /[(8 b+c+} \\
& +4 g+u)(8 b+c+4 g-u)(2 b+g)], \\
n= & {\left[\left(8 b^{2}-4 b c+32 b g-c^{2}+16 g^{2}+u^{2}\right)(2 b+g)\right] /[4(3 b+2 g)], } \\
q= & {\left[-\left(80 b^{3}+32 b^{2} c+24 b^{2} g-b c^{2}+16 b c g-40 b g^{2}+b u^{2}-2 c^{2} g\right.\right.} \\
& \left.\left.-16 g^{3}+2 g u^{2}\right) l\right] /[(8 b+c+4 g+u)(8 b+c+4 g-u)(2 b+g)], \\
l^{2}= & {\left[(8 b+c+4 g+u)(8 b+c+4 g-u)(2 b+g)^{2}(b+g)\right] /[8(3 b+2 g)] . }
\end{aligned}
$$

The invariant conic (3.2) is a parabola, where

$$
\begin{aligned}
& a_{10}=-3 b-g, \quad a_{01}=(2 l) /(2 b+g), \\
& a_{20}=-2(2 b+g)(b+g), \quad a_{11}=-4 l, \quad a_{02}=\left(-2 l^{2}\right) /[(2 b+g)(b+g)] .
\end{aligned}
$$

If $b^{2}+b g+s \neq 0$, then denote $\sqrt{g^{2}-4 s}=v$. We express $s=\left(g^{2}-v^{2}\right) / 4$ and replacing in $v_{2}=0$ one obtain $v_{2}=h_{1} \cdot h_{2}$, where $h_{1,2}=(2 b+g \pm v) a_{01}-4 l$.

Let $h_{1,2}=0$, then we get $a_{01}=(4 l) /(2 b+g \pm v)$. In this case we obtain the following series of conditions for the existence of a conic:

19)

$$
\begin{aligned}
a= & f=k=p=r=0, \quad m=\left(c^{2}-u^{2}\right) / 4, \quad s=\left(g^{2}-v^{2}\right) / 4, \\
d= & {\left[2 l \left(48 b^{2}+24 b c+48 b g \pm 40 b v+2 c^{2}+14 c g \pm 10 c v+11 g^{2} \pm 20 g v\right.\right.} \\
& \left.\left.-2 u^{2}+9 v^{2}\right)\right] /[(4 b+c+2 g+u \pm 2 v)(4 b+c+2 g-u \pm 2 v)(2 b+g \pm v)], \\
n= & {\left[\left(12 b g \pm 4 b v-c^{2}+2 c g \mp 2 c v+8 g^{2} \pm 8 g v+u^{2}\right)(2 b+g \pm v)\right] / } \\
& {[4(4 b+3 g \pm v)], } \\
q= & {\left[\left(48 b^{2} g \mp 16 b^{2} v+4 b c^{2}+16 b c g \mp 16 b c v\right.\right.} \\
& +52 b g^{2} \pm 32 b g v-4 b u^{2}-20 b v^{2}+5 c^{2} g \mp c^{2} v+8 c g^{2}-8 c v^{2} \\
& \left.\left.+14 g^{3} \pm 22 g^{2} v-5 g u^{2}+2 g v^{2} \pm u^{2} v \mp 6 v^{3}\right) l\right] /[(4 b+c+2 g \\
& +u \pm 2 v)(4 b+c+2 g-u \pm 2 v)(2 b+g \pm v)], \\
l^{2}= & {[(4 b+c+2 g+u \pm 2 v)(4 b+c+2 g-u \pm 2 v)(2 b} \\
& \left.+g \pm v)^{2}(g \pm v)\right] /[32(3 g \pm v+4 b)] .
\end{aligned}
$$

The invariant conic (3.2) is a parabola, where

$$
\begin{aligned}
& a_{10}=(-4 b-g \mp v) / 2, \quad a_{01}=(4 l) /(2 b+g \pm v), \\
& a_{20}=[-(g \pm v+2 b)(g \pm v)] / 2, \quad a_{11}=-4 l, \quad a_{02}=\left(-8 l^{2}\right) /[(2 b+g \pm v)(g \pm v)] .
\end{aligned}
$$

Theorem 3.1. The cubic differential system (1.1) has two parallel invariant straight lines and at least one invariant conic if and only if one of the following nineteen series of conditions 1)-19) holds. 


\section{Sufficient conditions for the existence of a centre}

Lemma 4.1. The following four series of conditions are sufficient conditions for the origin to be a centre for system (1.1):

i)

$$
\begin{aligned}
a & =f=k=p=r=0, \quad c=\left(-b^{2} s-2 l^{2}-2 s^{2}\right) /(b s), \\
d & =\left(-b^{2} s+l^{2}+s^{2}\right) /(b l), \quad g=\left[-\left(b^{2} s+l^{2}+s^{2}\right)\right] /(b s), \\
m & =\left(b^{2} l^{2} s+b^{2} s^{3}+l^{4}+2 l^{2} s^{2}+s^{4}\right) /\left(b^{2} s^{2}\right), \quad n=-3 s, \\
q & =\left(-2 b^{2} l^{2} s+b^{2} s^{3}-l^{4}-2 l^{2} s^{2}-s^{4}\right) /\left(b^{2} l s\right) .
\end{aligned}
$$

ii)

$$
\begin{aligned}
a & =f=k=p=r=0, \quad c=-b, \quad d=(b l) / s, \quad g=-b, \\
m & =\left(l^{2}+s^{2}\right) / s, \quad n=\left(l^{2}-2 s^{2}\right) / s, \quad q=-3 l .
\end{aligned}
$$

iii)

$$
\begin{aligned}
& a=f=k=p=r=0, \quad g=-b, \quad s=(m-n) / 3, \\
& d=\left(2 b m^{2}+5 b m n+2 b n^{2}-2 c m^{2}+c m n+c n^{2}\right) /[3 l(2 m+n)], \\
& q=\left(-2 m^{2}+m n+n^{2}\right) /(3 l), \quad l^{2}=\left(2 m^{2}-m n-n^{2}\right) / 9 .
\end{aligned}
$$

iv)

$$
\begin{aligned}
a & =f=k=p=r=0, \quad m=b g+g^{2}-\lambda, \quad s=(2 \lambda-n) / 3, \\
d & =\left(2 b^{2} n+5 b^{2} \lambda+3 b g n+6 b g \lambda+g^{2} n+g^{2} \lambda-n \lambda+2 \lambda^{2}\right) /[3 l(b+g)], \\
q & =\left(-b^{2} n+2 b^{2} \lambda+3 b g \lambda+g^{2} n+g^{2} \lambda+n^{2}-2 n \lambda\right) /(3 l), \\
l^{2} & =[(3 b(b+g)-n+2 \lambda)(n+\lambda)] / 9, \quad \lambda=(b-c+2 g)(b+g) .
\end{aligned}
$$

Proof. In each of the cases i)-iv) the first two Liapunov quantities vanish $L_{1}=$ $L_{2}=0$. The system (1.1) has four invaraint straight lines of which two are parallel and one invariant conic:

In the case i): $l_{1}=b s-x\left(l^{2}+s^{2}\right), l_{2}=b s-x\left(b^{2} s+l^{2}+s^{2}\right), l_{3,4}=$ $1+A_{1,2} x+l\left(b+A_{1,2}\right)^{-1} y$, where $A_{1,2}=\left[-l^{2}-s^{2}-b^{2} s \pm\left(b^{2} s\left(b^{2} s+2 l^{2}-2 s^{2}\right)+\right.\right.$ $\left.\left.\left(l^{2}+s^{2}\right)^{2}\right)^{1 / 2}\right] /(2 b s), f_{1}=b s^{2} x^{2}-b l s x y-\left(b^{2} s+l^{2}+s^{2}\right) x+b s$.

In the case ii): $l_{1,2}=2-b x \pm \sqrt{\left(b^{2} s-4 l^{2}-4 s^{2}\right) / s} x, l_{3,4}=1+A_{1,2} x+l(b+$ $\left.A_{1,2}\right)^{-1} y$, where $A_{1,2}=\left(-b \pm \sqrt{b^{2}-4 s}\right) / 2, f_{1}=s x^{2}-l x y-b x+1$.

In the case iii): $l_{1,2}=2+\left(c \pm \sqrt{c^{2}-4 m}\right) x, l_{3,4}=1+A_{1,2} x+l\left(b+A_{1,2}\right)^{-1} y$, where $A_{1,2}=\left(-3 b \pm \sqrt{9 b^{2}-12 m+12 n}\right) / 6, f_{1}=\left(2 m^{2}-m n-n^{2}\right) x^{2}-9 l(b+$ c) $y+3(2 m+n)(1-b x-l x y)$.

In the case iv): $l_{1}=1+(b+g) x, l_{2}=1+(c-b-g) x, l_{3,4}=1+A_{1,2} x+$ $l\left(b+A_{1,2}\right)^{-1} y$, where $A_{1,2}=\left(3 g \pm \sqrt{24 b c-24 b^{2}-72 b g+24 c g-39 g^{2}+12 n}\right) / 6$, $f_{1}=(\lambda+n)\left[(2 \lambda-n) x^{2}+3 g x+3\right]+3 l(2 \lambda-n) x y+9 l(b-c+2 g) y$.

By Theorem 2.3 in each of these cases the origin is a centre. 
Lemma 4.2. The following four series of conditions are sufficient conditions for the origin to be a centre for system (1.1):

i)

$$
\begin{aligned}
& a=f=k=l=p=q=r=0, \\
& c=-b(m+s) /(2 s), \quad g=-b, \quad n=-2 m .
\end{aligned}
$$

ii)

$$
\begin{aligned}
& a=f=k=p=r=l=0, \quad m=(c-b-g)(b+g), \\
& n=(c-b-2 g)(b+g), \quad q=(b+g) d, \\
& b^{2} g-b c g+3 b g^{2}-4 b s-c g^{2}+2 c s+2 g^{3}-5 g s=0 .
\end{aligned}
$$

iii)

$$
a=f=k=p=r=b=c=d=g=l=q=0, \quad m=-2 n .
$$

iv)

$$
\begin{aligned}
& a=f=k=p=r=d=q=l=0, \quad m=2 b^{2}, \\
& c=-3 b, \quad g=-2 b, \quad n=\left(-3 b^{2}\right) / 2 .
\end{aligned}
$$

Proof. In each of the cases i)-iv) the first Liapunov quantity vanish $L_{1}=0$. The system (1.1) has two parallel invaraint straight lines and two invariant conics:

In the case i): $l_{1,2}=4 s+\left(-b m-b s \pm \sqrt{b^{2}(m+s)^{2}-16 m s^{2}}\right) x, f_{1,2}=$ $2 s^{2} x^{2}-2 b s x+\left(d s \pm \sqrt{2 b^{2} s^{2}-2 b^{2} m s+d^{2} s^{2}+8 m s^{2}-8 s^{3}}\right) y+2 s$.

In the case ii): $l_{1}=1+(b+g) x, l_{2}=1+(c-b-g) x$,

$f_{1,2}=2 s x^{2}+2 g x+(b x+g x+1)\left(d \pm \sqrt{4 b g-4 c g+d^{2}+8 g^{2}-8 s}\right) y+2$.

If the case iii): $l_{1,2}=1 \pm x \sqrt{2 n}, f_{1,2}=s x^{2}-n y^{2} \pm y \sqrt{-4 n-2 s}+1$.

If the case iv):

$l_{1}=1-b x, \quad l_{2}=1-2 b x, \quad f_{1,2}=2 s x^{2} \pm 2 \sqrt{-2 s}(1-b x) y-b^{2} y^{2}-4 b x+2$.

By Theorem 2.2 in each of these cases the origin is a centre.

Lemma 4.3. The following two series of conditions are sufficient conditions for the origin to be a centre for system (1.1):

i)

$$
\begin{aligned}
& a=f=k=p=r=0, \quad c=-b, \quad g=(-3 b) / 2, \quad s=b^{2} / 2, \\
& l=(b d) / 6, \quad n=\left(-9 b^{2}+4 d^{2}\right) / 18, \quad q=-b d, \quad m=\left(9 b^{2}+8 d^{2}\right) / 36 .
\end{aligned}
$$

ii)

$$
\begin{aligned}
a & =f=k=p=r=0, \quad c=2(b+g), \quad d=(9 l) /(3 b+2 g), \\
m & =\left[36\left(3 b^{2}+6 b g+4 g^{2}\right) b g+32 g^{4}-81 l^{2}\right] /\left[9(3 b+2 g)^{2}\right], \\
n & =\left[-2\left(27 b^{3} g+54 b^{2} g^{2}+36 b g^{3}+8 g^{4}-81 l^{2}\right)\right] /\left[9(3 b+2 g)^{2}\right], \\
q & =(3 g l) /(3 b+2 g), \quad s=\left(2 g^{2}\right) / 9 .
\end{aligned}
$$


Proof. In the cases i) and ii) the system (1.1) has three invariant straight lines of which two are parallel and two invariant conics. The system (1.1) has a first integral of the form

$$
l_{1}^{\alpha_{1}} l_{2}^{\alpha_{2}} l_{3}^{\alpha_{3}} f_{1}^{\alpha_{4}} f_{2}^{\alpha_{5}}=C .
$$

In the case i) $l_{1,2}=6+(-3 b \pm i 2 \sqrt{2} d) x, l_{3}=6-3 b x+2 d y$,

$$
f_{1,2}=3 b^{2} x^{2}-b\left(3 a_{01}+2 d\right) x y-9 b x+2 d a_{01} y^{2}+6 a_{01} y+6,
$$

where $a_{01}=\left(4 d \pm \sqrt{18 b^{2}+16 d^{2}}\right) / 6$ and $\alpha_{1}=\alpha_{2}=1, \alpha_{3}=4, \alpha_{4}=\alpha_{5}=-1$.

In the case ii):

$$
l_{1,2}=3(3 b+2 g)+[3(3 b+2 g)(b+g) \pm \mu] x, \quad l_{3}=(3 b+2 g)(3+2 g x)+9 l y,
$$

$f_{1,2}=(6 b+2 g \pm 3 u)\left[2 g(3 b+2 g) x^{2}+18 l x y+2(3 b+2 g)^{2} y^{2}\right]+3 x(6 b-2 g \pm 3 u)(3 b+2 g)-$ $108 l y-18(3 b+2 g)$, where $\mu=\sqrt{(3 b+2 g)^{2}(3 b+g)^{2}+81 l^{2}}, u=2 \mu /[3(3 b+2 g)]$ and $\alpha_{1}=\alpha_{2}=\alpha_{4}=\alpha_{5}=1, \alpha_{3}=-4$.

Lemma 4.4. The following seven series of conditions are sufficient conditions for the origin to be a centre for system (1.1):

i)

$$
\begin{aligned}
a & =d=f=k=p=r=0, \quad b=\left[g\left(2 l^{2}-g^{2} s+4 s^{2}\right)\right] /\left[s\left(g^{2}-4 s\right)\right], \\
c & =\left[g\left(g^{2} s+2 l^{2}-4 s^{2}\right)\right] /\left[s\left(g^{2}-4 s\right)\right], \quad n=\left[2\left(4 s^{2}-g^{2} s-2 l^{2}\right)\right] /\left(g^{2}-4 s\right), \\
m & =\left(2 g^{2} l^{2}+g^{2} s^{2}-4 l^{2} s-4 s^{3}\right) /\left[s\left(g^{2}-4 s\right)\right], \quad q=-3 l .
\end{aligned}
$$

ii)

$$
\begin{aligned}
& a=f=k=p=r=0, \quad c=b+2 g, \quad d=\left[3\left(b g+g^{2}-m\right)(b+g)\right] / l, \\
& q=\left[3\left(b g+g^{2}-m\right)(b+g) g\right] /(2 l), \quad s=\left[\left(b g+g^{2}-m\right) g\right] /(2 b+g), \\
& n=2\left(b g+g^{2}-m\right), \quad l^{2}=\left[\left(b g+g^{2}-m\right)(2 b+g)(b+g)\right] / 2 .
\end{aligned}
$$

iii)

$$
\begin{aligned}
a= & f=k=p=r=0, \quad d=\left[\left(3 b c g-b^{2} g-3 b g^{2}-2 b m+2 b n+3 c g^{2}\right.\right. \\
& \left.\left.-2 g^{3}-4 g m+g n\right) \gamma+9(b-c+2 g) l^{2}\right] /(3 l \gamma), \\
q= & {\left[\left(m-n-b g-g^{2}\right)\right.} \\
& \left.\cdot\left(b g+g^{2}-4 m-2 n\right) \gamma+9 l^{2}\left(2 b g+2 g^{2}-2 m-n\right)\right] /(9 l \gamma), \\
s= & {\left[\left(2 b g+2 g^{2}-2 m-n\right)\left(b g+g^{2}-m+n\right)\right] /(3 \gamma), } \\
3 b^{3} g-9 b^{2} c g+11 b^{2} g^{2}+6 b^{2} m-6 b^{2} n-18 b c g^{2}+13 b g^{3}+23 b g m & \\
& -8 b g n-9 c g^{3}+5 g^{4}+17 g^{2} m-2 g^{2} n+18 l^{2}-4 m^{2}+2 m n+2 n^{2}=0, \\
b^{3} g^{2}-5 b^{2} c g^{2}+4 b^{2} g^{3}+7 b^{2} g m-4 b^{2} g n-10 b c g^{3}+b c g m+2 b c g n & \\
& +5 b g^{4}+21 b g^{2} m-6 b g^{2} n-8 b m^{2}+4 b m n+4 b n^{2}-5 c g^{4}+c g^{2} m \\
& +2 c g^{2} n+4 c m^{2}-2 c m n-2 c n^{2}+2 g^{5}+14 g^{3} m-2 g^{3} n-16 g m^{2} \\
& +2 g m n+5 g n^{2}=0, \\
\gamma= & 3 b^{2}-3 b c+7 b g-3 c g+4 g^{2}+2 m+n .
\end{aligned}
$$


iv)

$$
\begin{aligned}
& a=f=k=p=r=b=c=g=l=q=0, \\
& s=\left[m\left((m+2 n)^{2}-d^{2}(m+n)\right)\right] /(m+2 n)^{2} .
\end{aligned}
$$

v)

$$
\begin{aligned}
a & =f=k=p=r=l=0, \quad c=-3 b, \quad g=-2 b, \\
m & =2 b^{2}, \quad q=-b d, \quad s=\left[\left(b^{2}+n\right) b^{2} d^{2}\right] /\left(3 b^{2}+2 n\right)^{2} .
\end{aligned}
$$

vi)

$$
\begin{aligned}
a & =f=k=p=r=0, \quad c=(4 b+5 g) / 2, \quad m=g(b+g), \\
l & =\left[6 d(2 b+g)(b+g)^{2}\right] /\left[36(b+g) b+4 d^{2}+9 g^{2}\right], \\
n & =\left[-3\left(18 b g-4 d^{2}+9 g^{2}\right)(2 b+g)(b+g)\right] /\left[2\left(36(b+g) b+4 d^{2}+9 g^{2}\right)\right], \\
s & =\left(18 b g-4 d^{2}+9 g^{2}\right)^{2} /\left[36\left(36(b+g) b+4 d^{2}+9 g^{2}\right)\right], \\
q & =\left[-d\left(18 b g-4 d^{2}+9 g^{2}\right)(b+g)\right] /\left[36(b+g) b+4 d^{2}+9 g^{2}\right] .
\end{aligned}
$$

vii)

$$
\begin{aligned}
& a=f=k=p=r=d=l=q=0, \\
& g=\left(b a_{02}+c a_{02}+2 b^{3}+3 b^{2} c+b c^{2}-b m-3 b n-c n\right) / a_{02}, \\
& s=\left(m a_{02}+n a_{02}+2 b^{2} m+2 b^{2} n+b c m+b c n-m n-n^{2}\right) / a_{02} .
\end{aligned}
$$

Proof. In each of the cases i)-vii) the system (1.1) has two parallel invariant straight lines and one invariant conic. In the cases i)-vi) the system (1.1) has a Darboux integrating factor of the form

$$
\mu=l_{1}^{\alpha_{1}} l_{2}^{\alpha_{2}} f_{1}^{\alpha_{3}} .
$$

In the case i): $l_{1,2}=\left(g^{3} s+2 g l^{2}-4 g s^{2} \pm A\right) x+2 g^{2} s-8 s^{2}, f_{1}=s x^{2}-l x y+g x+1$,

$$
A=\left(g^{6} s^{2}-4 g^{4} l^{2} s-12 g^{4} s^{3}+4 g^{2} l^{4}+32 g^{2} l^{2} s^{2}+48 g^{2} s^{4}-64 l^{2} s^{3}-64 s^{5}\right)^{1 / 2}
$$

and $\alpha_{1,2}=\left[g l^{2}\left(g A \mp\left(g^{4} s-2 g^{2} l^{2}-6 g^{2} s^{2}+8 l^{2} s+8 s^{3}\right)\right)\right] /\left[A\left(2 g^{2} l^{2}+g^{2} s^{2}-4 l^{2} s-\right.\right.$ $\left.\left.4 s^{3}\right)\right], \alpha_{3}=-3$.

In the case ii): $l_{1,2}=2+\left(b+2 g \pm \sqrt{b^{2}+4 b g+4 g^{2}-4 m}\right) x, f_{1}=g\left(b g+g^{2}-\right.$ $m) x^{2}+g l x y+g(2 b+g) x+2 l y+2 b+g$ and $\alpha_{1,2}=b g+g^{2} \mp\left(b^{2} g+3 b g^{2}-2 b m+\right.$ $\left.2 g^{3}-2 g m\right) /\left[2 m \sqrt{b^{2}+4 b g+4 g^{2}-4 m}\right], \alpha_{3}=-3$.

In the case iii): $l_{1,2}=2+\left(c \pm \sqrt{c^{2}-4 m}\right) x, f_{1}=s x^{2}+\left((b+g) a_{01}-l\right) x y+$ $g x+a_{01} y+1=0, a_{01}=(3 l(b-c+2 g)) / \gamma$ and $\alpha_{1,2}=\left[\left(b g+g^{2}\right) \sqrt{c^{2}-4 m} \mp(b c g-\right.$ $\left.\left.4 b m+c g^{2}+2 c m-6 g m\right)\right] /\left[2 m \sqrt{c^{2}-4 m}\right], \alpha_{3}=-3$.

In the case iv): $l_{1,2}=1 \pm x \sqrt{-m}, f_{1}=m\left[d^{2}(m+n)-(m+2 n)^{2}\right] x^{2}-(m+$ $2 n)^{2}(m+n) y^{2}-2 d(m+2 n)(m+n) y-(m+2 n)^{2}$ and

$$
\alpha_{1}=\alpha_{2}=(-2 m-n) /[2(m+n)], \quad \alpha_{3}=(-m-2 n) /[2(m+n)] .
$$


In the case v): $l_{1}=1-b x, l_{2}=1-2 b x, f_{1}=b^{2} d^{2}\left(b^{2}+n\right) x^{2}-2\left(3 b^{2}+2 n\right)\left(b^{2}+\right.$ $n) b d x y-2\left(3 b^{2}+2 n\right)^{2} b x+\left(3 b^{2}+2 n\right)^{2}\left(b^{2}+n\right) y^{2}+2\left(3 b^{2}+2 n\right)\left(b^{2}+n\right) d y+\left(3 b^{2}+2 n\right)^{2}$ and $\alpha_{1}=-2, \alpha_{2}=(-n) /\left[2\left(b^{2}+n\right)\right], \alpha_{3}=\left(-3 b^{2}-2 n\right) /\left[2\left(b^{2}+n\right)\right]$.

In the case vi): $l_{1}=2(b+g) x+1, l_{2}=2+g x, f_{1}=\left(18 b g-4 d^{2}+9 g^{2}\right)^{2} x^{2}-$ $24 d\left(18 b g-4 d^{2}+9 g^{2}\right)(b+g) x y+144(b+g)^{2} d^{2} y^{2}+(36 g x+24 d y+36)\left(9(2 b+g)^{2}+4 d^{2}\right)$ and $\alpha_{1}=0, \alpha_{2}=-2, \alpha_{3}=(-3) / 2$.

Suppose for (1.1) conditions vii) hold. In this case the system (1.1) has a Darboux first integral of the form $l_{1}^{\alpha_{1}} l_{2}^{\alpha_{2}} f_{1}^{\alpha_{3}}=C$, where

$$
\begin{gathered}
l_{1,2}=2+x\left(c \pm \sqrt{c^{2}-4 m}\right), \quad f_{1}=\left(a_{02}+2 b^{2}+b c-n\right) x^{2}+a_{02} y^{2}-2 b x+1 \\
\text { and } \alpha_{1}=n \sqrt{c^{2}-4 m}+2 b m-c n, \alpha_{2}=n \sqrt{c^{2}-4 m}-2 b m+c n, \alpha_{3}=m \sqrt{c^{2}-4 m} .
\end{gathered}
$$

Lemma 4.5. The following five series of conditions are sufficient conditions for the origin to be a centre for system (1.1):

i)

$$
\begin{aligned}
& a=f=k=p=r=q=g=s=0, \quad d=(3 l) / b, \quad n=\left(2 l^{2}\right) / b^{2}, \\
& 4 l^{4}+2 l^{2} b^{2}\left(2 b c-c^{2}+4 m\right)+b^{4} m\left(4 b^{2}-c^{2}+4 m\right)=0 .
\end{aligned}
$$

ii)

$$
\begin{aligned}
a= & f=k=p=r=0, \quad q=b d+d g-3 l \\
s= & {[(b d+d g-3 l)(3 l-b d)] / d^{2} } \\
n= & {\left[\left((5 b-c+4 g)(b+c)(b+g) d^{3}\right.\right.} \\
& -3 l d^{2}\left(20 b^{2}+10 b c+26 b g-c^{2}+8 c g+8 g^{2}\right)+27 l^{2} d(11 b+2 c+7 g) \\
& \left.\left.-486 l^{3}\right)(b d-3 l)\right] /\left[(3 b d+2 d g-9 l)^{2} d^{2}\right], \\
m= & {\left[-\left(b^{2} d^{2}+b c d^{2}+b d^{2} g+6 b d l+c d^{2} g-3 c d l+3 d g l-27 l^{2}\right)\left(b^{2} d^{2}-2 b c d^{2}\right.\right.} \\
& \left.\left.+b d^{2} g+6 b d l-c d^{2} g+6 c d l+3 d g l-27 l^{2}\right)\right] /\left[(2 d g-9 l+3 b d)^{2} d^{2}\right], \\
2(3 b d+2 d g-9 l)^{2} d^{3}+9\left(4 b^{2} d+b c d+3 b d g-12 b l+c d g\right. & \\
& -3 c l-3 g l)(b d+c d-9 l)(b d-3 l)=0 .
\end{aligned}
$$

iii)

$$
\begin{aligned}
a= & f=k=p=r=0, \quad m=-(9 b-c+7 g)(9 b-2 c+7 g), \\
d= & {\left[2 l\left(27 b^{2}-11 b c+39 b g+c^{2}-8 c g+14 g^{2}\right)\right] /[(8 b-c+6 g)} \\
& \cdot(5 b-c+4 g)(b+g)], \quad s=-(6 b-c+5 g)(6 b-c+4 g), \\
n= & 30 b^{2}-12 b c+44 b g+c^{2}-9 c g+16 g^{2}, \\
q= & {\left[l\left(-66 b^{2}+17 b c-108 b g-c^{2}+14 c g-44 g^{2}\right)\right] /\left(40 b^{2}\right.} \\
& \left.-13 b c+62 b g+c^{2}-10 c g+24 g^{2}\right), \\
l^{2}= & {[(c-8 b-6 g)(5 b-c+4 g)(3 b-c+3 g)(b+g)] / 2, }
\end{aligned}
$$




$$
\begin{aligned}
& 738 b^{3}-309 b^{2} c+1710 b^{2} g+43 b c^{2}-476 b c g+1318 b g^{2}-2 c^{3} \\
& \quad+33 c^{2} g-183 c g^{2}+338 g^{3}=0 .
\end{aligned}
$$

iv)

$$
\begin{aligned}
a & =f=k=p=r=0, \quad c=\left(-9 b^{2} d^{2}-2 d^{4}+81 l^{2}\right) /[9 d(b d-3 l)], \\
m & =\left[2\left(27 b^{2} d^{2} l-b d^{5}-162 b d l^{2}-d^{5} g+3 d^{4} l+243 l^{3}\right)\right] /\left[9 d^{2}(b d-3 l)\right], \\
n & =[9 l(3 l-b d)] / d^{2}, \quad q=b d+d g-3 l, \quad s=[(3 l-b d-d g)(b d-3 l)] / d^{2} .
\end{aligned}
$$

v)

$$
\begin{aligned}
a & =f=k=p=r=0, \quad c=(9 b+7 g) / 2, \\
m & =\left[8(3 b+2 g)^{2}(b+g)\right] /(17 b+11 g), \\
d & =[-2 l(27 b+17 g)] /[(5 b+3 g)(b+g)], \\
n & =\left[\left(33 b^{2}-2 b g-15 g^{2}\right)(b+g)\right] /[4(17 b+11 g)], \\
s & =[-3(3 b+g)(b+g)] / 4, \quad q=[-l(69 b+43 g)] /(5 b+3 g), \\
l^{2} & =\left[-(5 b+3 g)^{2}(3 b+g)(b+g)^{2}\right] /[16(17 b+11 g)] .
\end{aligned}
$$

Proof. In the cases i)-v) the system (1.1) has three invariant straight lines of which two are parallel and one invariant conic. The system (1.1) has a Darboux integrating factor of the form

$$
\mu=l_{1}^{\alpha_{1}} l_{2}^{\alpha_{2}} l_{3}^{\alpha_{3}} f_{1}^{\alpha_{4}} .
$$

In the case i $): l_{1,2}=2+x\left(c \pm \sqrt{c^{2}-4 m}\right), l_{3}=b+l y, f_{1}=2\left(2 b^{4}-b^{3} c+\right.$ $\left.b^{2} m+l^{2}\right)(2 l x y+b x)+b^{2}\left(4 b^{4}-b^{2} c^{2}+4 b^{2} m+4 l^{2}\right) y^{2}-b^{2}(2 b-c)(b+2 l y)$ and $\alpha_{1}=\alpha_{2}=0, \alpha_{3}=-\left(2 b^{4}+b^{3} c-b^{2} c^{2}+3 b^{2} m+3 l^{2}\right) /\left(2 b^{4}-b^{3} c+b^{2} m+l^{2}\right)$, $\alpha_{4}=-\left[(2 b-c)^{2} b^{2}\right] /\left[2\left(2 b^{4}-b^{3} c+b^{2} m+l^{2}\right)\right]$.

In the case ii): $l_{1}=d(3 b d+2 d g-9 l)+x\left(b^{2} d^{2}+b c d^{2}+b d^{2} g+6 b d l+c d^{2} g-3 c d l+\right.$ $\left.3 d g l-27 l^{2}\right), l_{2}=d(3 b d+2 d g-9 l)+x\left(2 b c d^{2}-b^{2} d^{2}-b d^{2} g-6 b d l+c d^{2} g-6 c d l-3 d g l+\right.$ $\left.27 l^{2}\right), l_{3}=3 d+3(3 l-b d) x+d^{2} y, f_{1}=(b d+c d-9 l)\left[3(b d+d g-3 l)(b d-3 l) x^{2}-2(b d+\right.$ $\left.d g-3 l) d^{2} x y+9 l(b d-3 l) y^{2}\right]+3 d^{2}\left(2 b^{2} d-b c d+b d g-6 b l-c d g+3 c l+3 g l\right) x-(2 d g-$ $9 l+3 b d)(2 d y+3) d^{2}$ and $\alpha_{1}=\alpha_{3}=0, \alpha_{2}=\left[d\left(3 b c d-6 b^{2} d-5 b d g+18 b l+c d g-\right.\right.$ $9 c l+9 g l)] /\left[2\left(b^{2} d^{2}-2 b c d^{2}+b d^{2} g+6 b d l-c d^{2} g+6 c d l+3 d g l-27 l^{2}\right)\right], \alpha_{4}=(-3) / 2$.

In the case iii): $l_{1}=1+(9 b-c+7 g) x, l_{2}=1-(9 b-2 c+7 g) x, l_{3}=$ $(7 b-c+5 g)[1+(6 b-c+5 g) x]+l y, f_{1}=(5 b-c+4 g)\left[(6 b-c+4 g)(b+g) x^{2}-(5 b-\right.$ $\left.c+3 g) x+(8 b-c+6 g)(b+g) y^{2}-1\right]+2 l(b+g) x y+2 l y$ and $\alpha_{1}=(-3) / 2, \alpha_{2}=$ $1, \alpha_{3}=2, \alpha_{4}=(-5) / 2$.

In the case iv): $l_{1,2}=2+\left(c \pm \sqrt{c^{2}-4 m}\right) x, l_{3}=3 d+3(3 l-b d) x+d^{2} y$, $f_{1}=18 l(b d-3 l)^{2} x^{2}+2 l d^{4} y^{2}-3 d\left(b^{2} d^{2}-9 l^{2}\right) x+(b d-3 l)\left(3 d^{2}+2 d^{3} y-12 l d^{2} x y\right)$ and $\alpha_{1}=\alpha_{2}=\alpha_{3}=\alpha_{4}=-1$.

In the case v): $l_{1,2}=4+(9 b+7 g \pm(5 b+3 g) \sqrt{(9 b+3 g) /(17 b+11 g)}) x$, $l_{3}=(2+3(b+g) x)(5 b+3 g)+4 l y, f_{1}=(17 b+11 g)\left[(3 b+g)(b+g)^{2} x^{2}+8 l(b+\right.$ g) $\left.x y+\left(b^{2}-g^{2}\right) x+8 l y-2(b+g)\right]-(5 b+3 g)^{2}(b+g)^{2} y^{2}$ and $\alpha_{1}=\alpha_{2}=(-3) / 2$, $\alpha_{3}=2, \alpha_{4}=(-5) / 2$. 


\section{Solution of the problem of the centre for cubic differential system with two parallel invariant straight lines and at least one invariant conic}

Theorem 5.1. $\left(l_{j}=1+A_{j} x+B_{j} y, j=1,2, l_{1} \| l_{2}, f ; L=3\right)$, where $f=0$ is an invariant conic of the form (3.2), is ILC for system (1.1), i.e. if the cubic system (1.1) has two parallel invariant straight lines and one invariant conic then the order of the weak focus $O(0,0)$ is at most 3 .

Proof. To prove the theorem, we compute the first three Liapunov quantities $L_{1}, L_{2}, L_{3}$ in each series of conditions 1)-19) using the algorithm described in [22]. In the expressions for $L_{j}$ we will neglect with denominators and non-zero factors.

In the case 1) the first Liapunov quantity is $L_{1}=2 b^{2} s+b g n+5 b g s+g^{2} n+$ $3 g^{2} s+2 l^{2}-2 n s-4 s^{2}$. From $L_{1}=0$ we find $l^{2}$ and replacing into the expression for $L_{2}$, we obtain $L_{2}=f_{1} f_{2} f_{3} f_{4}$, where $f_{1}=n+3 s, f_{2}=b+g, f_{3}=2 b s+g n+4 g s$, $f_{4}=2 b^{2} s+b g n+5 b g s+g^{2} n+3 g^{2} s-2 n s-4 s^{2}$.

If $f_{1}=0$, then we are in the conditions of Lemma $\left.4.1, \mathrm{i}\right)$, if $f_{2}=0$, then Lemma 4.1, ii), if $f_{3}=0$, then Lemma $\left.4.4, \mathrm{i}\right)$ and if $f_{4}=0$, then $I_{3}=0$.

In the cases 2) and 4) the first Liapunov quantity vanishes. We are in the conditions of Lemma 4.2, i) and Lemma 4.2, ii) respectively.

In the cases 3) and 5) we have $L_{1}=q \neq 0$ and $L_{1}=b d+d g-q \neq 0$, respectively. Therefore the origin is a focus.

In the case 6) the first Liapunov quantity is $L_{1}=2 b^{3} g+5 b^{2} g^{2}-2 b^{2} m+$ $4 b g^{3}-3 b g m+g^{4}-g^{2} m-2 l^{2}$. From $L_{1}=0$ we find $l^{2}$ and replacing into the expression for $L_{2}$, we obtain $L_{2}=3 b^{2} g+6 b g^{2}-3(b+g) m-d l+3 g^{3}$. If $L_{2}=0$, then Lemma 4.4, ii).

In the case 7) the first Liapunov quantity is $L_{1}=3 b^{3} g-9 b^{2} c g+11 b^{2} g^{2}+$ $6 b^{2} m-6 b^{2} n-18 b c g^{2}+13 b g^{3}+23 b g m-8 b g n-9 c g^{3}+5 g^{4}+17 g^{2} m-2 g^{2} n+18 l^{2}-$ $4 m^{2}+2 m n+2 n^{2}$. From $L_{1}=0$ we find $l^{2}$ and replacing into the expression for $L_{2}$, we obtain $L_{2}=f_{1} f_{2} f_{3}$, where $f_{1}=b+g, f_{2}=b^{2}-b c+2 b g-c g+g^{2}+m$, $f_{3}=b^{3} g^{2}-5 b^{2} c g^{2}+4 b^{2} g^{3}+7 b^{2} g m-4 b^{2} g n-10 b c g^{3}+b c g m+2 b c g n+5 b g^{4}+$ $21 b g^{2} m-6 b g^{2} n-8 b m^{2}+4 b m n+4 b n^{2}-5 c g^{4}+c g^{2} m+2 c g^{2} n+4 c m^{2}-2 c m n-$ $2 c n^{2}+2 g^{5}+14 g^{3} m-2 g^{3} n-16 g m^{2}+2 g m n+5 g n^{2}$.

If $f_{1}=0$, then Lemma 4.1, iii), if $f_{2}=0$, then Lemma 4.1,iv) and if $f_{3}=0$, then Lemma 4.4, iii).

In the case 8) the first Liapunov quantity vanishes, then Lemma 4.4 vii).

In the case 9) the first Liapunov quantity is $L_{1}=l^{2}+s^{2}-b^{2} s$. From $L_{1}=0$ we find $l^{2}$ and replacing into the expression for $L_{2}$, we obtain $L_{2}=(3 b+c) b$. If $c=-3 b$, then Lemma 4.1,i) $\left(l^{2}+s^{2}-b^{2} s=0\right)$ and if $b=0$, then $I_{3}=0$.

In the case 10) the vanishing of the first Liapunov quantity gives $m=\left(b^{2} s-\right.$ $\left.l^{2}-s^{2}\right) /\left(b^{2}-s\right)$. Then $L_{2}=\left(b^{4}-2 b^{2} s+l^{2}+s^{2}\right) b^{2}$. If $b=0$, then Lemma 4.1, ii $)$ $(b=0)$, if $b^{4}-2 b^{2} s+l^{2}+s^{2}=0$, then $I_{3}=0$.

In the case 11) the first Liapunov quantity is $L_{1}=b$. If $b=0$ and $m=-2 n$, then Lemma 4.2 , iii). If $b=0$ and $m+2 n \neq 0$, then Lemma 4.4 , iv). 
In the case 12) the vanishing of the first Liapunov quantity gives

$$
d=\left(g a_{01}^{3}+6 l a_{01}^{2}-2 b^{2} g a_{01}-b g^{2} a_{01}+4 b g l+2 g^{2} l\right) /\left[a_{01}\left(g a_{01}+4 l\right)\right] .
$$

The second Liapunov quantity is $L_{2}=f_{1} f_{2} f_{3}$, where $f_{1}=g, f_{2}=a_{01}^{3}+$ $4 b^{2} a_{01}+2 b g a_{01}-8 b l-4 g l, f_{3}=3 b+2 g$.

If $f_{1}=0$, then Lemma 4.4, iii) $(g=0)$, if $f_{2}=0$, then Lemma 4.5, ii) ( $c=$ $2(b+g))$. Assume that $f_{1}, f_{2} \neq 0$ and let $f_{3}=0$. Then $g=(-3 b) / 2$ and $L_{3}=h_{1} h_{2}$, where $h_{1}=a_{01}^{3}+b^{2} a_{01}-2 b l, h_{2}=2 b a_{01}^{2}-16 l a_{01}-b^{3}$. If $h_{1}=0$, then Lemma 4.5 , ii) $(c=-b, g=(-3 b) / 2)$ and if $h_{2}=0$, then Lemma $\left.4.3 \mathrm{i}\right)$.

In the case 13) we calculate the first Liapunov quantity and find that $L_{1}=$ $3 b+c$. If $L_{1}=0$ and $3 b^{2}+2 n=0$, then Lemma 4.2 , iv). If $L_{1}=0$ and $3 b^{2}+2 n \neq 0$, then Lemma 4.4, v).

In the case 14) the vanishing of the first Liapunov quantity gives $c=[(4 b+$ $\left.5 g)(2 b+g)(b+g)^{2} a_{01}^{2}+4 l(2 b+g)(b+g)^{2} a_{01}-4 l^{2}\left(a_{01}^{2}+(2 b+g)^{2}\right)\right] /\left[2(2 b+g)(b+g)^{2} a_{01}^{2}\right]$. The second Liapunov quantity looks $L_{2}=f_{1} f_{2} f_{3} f_{4} f_{5}$, where $f_{1}=b a_{01}+g a_{01}-$ $2 l, f_{2}=b a_{01}+g a_{01}+2 l, f_{3}=l a_{01}^{2}-(2 b+g)(b+g)^{2} a_{01}+l(2 b+g)^{2}, f_{4}=$ $b+g, f_{5}=\left(4 l^{2}+3(2 b+g)^{2}(b+g)^{2}\right) a_{01}^{2}-4 l(2 b+g)(b+g)^{2} a_{01}+4 l^{2}(2 b+g)^{2}$.

If $f_{1}=0$, then Lemma 4.5 ,ii) $(d=(3 l) /(b+g))$, if $f_{2}=0$, then Lemma 4.5 , iv $)$ $(d=(-3 l) /(b+g))$, if $f_{3}=0$, then Lemma 4.4, vi), if $f_{4}=0$, then $I_{3}=0$. The resultant of $f_{5}$ and $L_{3}$ by $a_{01}$ is not equal to zero and the obtained system of equations $f_{5}=0$ and $L_{3}=0$ has no real solutions, i.e. in this case the origin $O(0,0)$ is a focus.

In the case 15) the first Liapunov quantity is $L_{1}=g_{1} g_{2}$, where

$$
g_{1}=14 b^{2}-b c+14 b g \pm 3 b u-c g+4 g^{2} \pm g u, \quad g_{2}=10 b-c+6 g \pm u .
$$

If $g_{1}=0$ and $3 b+g=0$, then Lemma 4.3, ii), if $g_{1}=0,3 b+g \neq 0$, then Lemma 4.5 , ii) $(d=(3 l) /(2 b+g))$, if $g_{2}=0$, then $I_{3}=0$.

In the case 16) the first Liapunov quantity is $L_{1}=g_{1} g_{2}$, where

$$
\begin{gathered}
g_{1}=8 b v-c g-c v+g^{2} \mp g u+4 g v \pm 3 u v+3 v^{2}, \quad g_{2}=6 b-c+4 g \pm u+2 v . \\
\text { If } g_{1}=0, v=g=0, \text { then Lemma 4.5, i). If } g_{1}=0, v=g / 3, c=2(b+g),
\end{gathered}
$$
then Lemma 4.3 , ii). If $g_{1}=0,3 v-g \neq 0$, then Lemma 4.5 , ii).

Assume that $g_{1} \neq 0$ and let $g_{2}=0$. Then $\pm u=-6 b+c-4 g-2 v$ and $L_{2}=12 b-2 c+9 g+v$. From $L_{2}=0$ we have $v=-12 b+2 c-9 g$ and calculate $L_{3}$. The third Liapunov quantities is $L_{3}=738 b^{3}-309 b^{2} c+1710 b^{2} g+43 b c^{2}-476 b c g+$ $1318 b g^{2}-2 c^{3}+33 c^{2} g-183 c g^{2}+338 g^{3}$.

If $L_{3}=0$, then Lemma 4.5, iii).

In the case 17) the first Liapunov quantity is $L_{1}=g_{1} g_{2}$, where $g_{1}=-8 b v-c g+c v+g^{2} \mp g u-4 g v \mp 3 u v+3 v^{2}, \quad g_{2}=6 b-c+4 g \pm u-2 v$.

If $g_{1}=0, v=g=0$, then Lemma 4.5, i). If $g_{1}=0, v=-g / 3, c=2(b+g)$, then Lemma 4.3 , ii). If $g_{1}=0,3 v+g \neq 0$, then Lemma 4.5, ii).

Assume that $g_{1} \neq 0$ and let $g_{2}=0$. Then $\pm u=-6 b+c-4 g+2 v$ and $L_{2}=12 b-2 c+9 g-v$. From $L_{2}=0$ we obtain $v=12 b-2 c+9 g$. Then $L_{3}=$ $738 b^{3}-309 b^{2} c+1710 b^{2} g+43 b c^{2}-476 b c g+1318 b g^{2}-2 c^{3}+33 c^{2} g-183 c g^{2}+338 g^{3}$. 
If $L_{3}=0$, then Lemma 4.5, iii).

In the case 18) the first Liapunov quantity is $L_{1}=g_{1} g_{2}$, where

$$
g_{1}=28 b^{2}+4 b c+28 b g+c^{2}+8 g^{2}-u^{2}, \quad g_{2}=5 b+3 g .
$$

If $g_{1}=0$, then Lemma 4.5 , iv $)(d=(3 l) /(2 b+g))$, if $g_{2}=0$, then $I_{3}=0$. In the case 19) the first Liapunov quantity is $L_{1}=g_{1} g_{2}$, where

$$
g_{1}=c^{2} \pm 8 b v-2 c g \pm 2 c v+g^{2} \pm 4 g v-u^{2}+3 v^{2}, \quad g_{2}=3 b+2 g \pm v .
$$

If $g_{1}=0$, then Lemma 4.5,iv). Assume that $g_{1} \neq 0$ and let $g_{2}=0$. Then $\pm v=$ $-3 b-2 g$ and $L_{2}=9 b-2 c+7 g$. From $L_{2}=0$ we get $c=(9 b+7 g) / 2$ and calculate $L_{3}$. The third Liapunov quantity is $L_{3}=225 b^{3}+345 b^{2} g+171 b g^{2}-68 b u^{2}+27 g^{3}-44 g u^{2}$. If $L_{3}=0$, then Lemma $4.5, \mathrm{v}$ ).

\section{References}

[1] V. V. Amel'kin, N. A. Lukashevich and A. P. Sadovsky, Non-linear oscillations in the systems of second order, Belarusian University Press, Belarus, 1982 (Russian).

[2] N. N. Bautin, On the number of limit cycles which appear with the variation of coefficients from an equilibrium position of focus or center type, Mat. Sb. 30 (72) (1952), 181-196; Amer. Math. Soc. Transl. 100 (1954), 397-413.

[3] Y.L. Bondar and A.P. Sadovskii, Variety of the center and limit cycles of a cubic system, which is reduced to Lienard form, Bull. Acad. Sci. of Moldova, Mathematics 46 (2004), no. 3, 71-90.

[4] J. Chavarriga, H. Giacomini, J. Gine, J. Llibre, On the integrability of twodimensional flows, J. Differential Equations 157 (1999), no. 1, 163-182.

[5] J. Chavariga and J. Giné, Integrability of cubic systems with degenerate infinity, Differential Equations and Dynamical Systems 6 (1998), no. 4, 425-438.

[6] J. Chavarriga, H. Giacomini, J. Giné, J. Llibre, Darboux integrability and the inverse integrating factor, J. Differential Equations 194 (2003), no. 1, 116-139.

[7] L. A. Cherkas, V. G. Romanovskii and H. Zoladek, The centre conditions for a certain cubic system, Differential Equations and Dynamical Systems 5 (1997), no. 3-4, 299302.

[8] C. Christopher, J. Llibre, C. Pantazi, X. Zhang, Darboux integrability and invariant algebraic curves for planar polynomial systems, J. Phys. A 35 (2002), no. 10, 24572476 .

[9] D. Cozma and A. Subă, Partial integrals and the first focal value in the problem of centre, NoDEA: Nonlinear Differential Equations and Applications 2 (1995), no. 1, $21-34$.

[10] D. Cozma and A. Şubă, The solution of the problem of center for cubic differential systems with four invariant straight lines, Scientific Annals of the "Al.I.Cuza" University (Romania), Mathematics, vol. XLIV (1998), s.I.a, 517-530.

[11] D. Cozma and A. Şubă, Solution of the problem of the centre for a cubic differential system with three invariant straight lines, Qualitative Theory of Dynamical Systems 2 (2001), no. 1, 129-143. 
[12] G. Darboux, Mémoire sur les équations différentielles algébriques du premier ordre et du premier degré (Mélanges), Bull. Sci. Math. 2-ème série 2 (1878) 60-96, 123-144, 151-200.

[13] H. Dulac, Détermination et intégration d'une certaine classe d'équations différentielles ayant pour point singulier un centre, Bull. Sciences Math. Sér. (2) 32 (1) (1908), 230-252.

[14] R. E. Kooij, Cubic systems with four line invariants, including complex conjugated lines, Differential Equations Dynam. Systems 4 (1996), no. 1, 43-56.

[15] G. Korn, T. Korn, Mathematical handbook, McGraw-Hill Book Company, 1968.

[16] A. M. Liapunov, General problem of stability of motion, Gostekhizdat, Moscow, 1950 (Russian).

[17] N. G. Lloyd, J. M. Pearson, V. G. Romanovsky, Computing integrability conditions for a cubic differential system, Computers Math. Applic. 32 (1996), no. 10, 99-107.

[18] V. G. Romanovskii and A. Şubă, Center of some cubic systems, Annals of Differential Equations (China) 17 (2001), no. 4, 363-376.

[19] A.P. Sadovskii, On center conditions for one cubic system of differential equations, Diff. Equations 36 (2000), no. 1, 98-102 (Russian).

[20] K. S. Sibirskii, On the number of limit cycles in the neighborhood of a singular point, Differentsial'nye Uravneniya 1 (1965), 51-66 (Russian).

[21] A. Şubă and D. Cozma, Solution of the problem of center for cubic differential systems with three invariant straight lines in generic position, Qualitative Theory of Dynamical Systems 6 (2005), 45-58.

[22] A. Şubă, Partial integrals, integrability and the center problem, Differ. Uravn. 32 (1996), no. 7, 880-888; translation in Differential Equations 32 (1996), no. 7, 884-892 (1997).

[23] H. Żołądek, On certain generalization of the Bautin's theorem, Nonlinearity 7 (1994), 273-279.

[24] H. Żołądek, The classification of reversible cubic systems with center, Topol. Meth. in Nonlin. Analysis 4 (1994), 79-136.

Dumitru Cozma

Department of Mathematics

Tiraspol State University

5 Gh. Iablocichin str.

Chişinău, MD-2069

Moldova

e-mail: dcozma@gmail.com

Received: 24 April 2008.

Accepted: 2 October 2008. 